\title{
Evaluación de la respuesta antioxidante en Chiton granosus Frembly, 1928 (Mollusca: Polyplacophora) a contaminantes oxidativos
}

\author{
Evaluation of the antioxidant response of Chiton granosus Frembly, 1928 (Mollusca: \\ Polyplacophora) to oxidative pollutants
}

\author{
María E. Hidalgo ${ }^{1}$, Ernesto Fernández ${ }^{2}$, Alicia Cabello ${ }^{3}$, Cristian Rivas ${ }^{3}$, Flavia Fontecilla ${ }^{3}$, \\ Lorena Morales ${ }^{3}$, Alvaro Aguirre $^{3}$ y Enrique Cabrera ${ }^{1}$ \\ ${ }^{1}$ Facultad de Ciencias, Universidad de Valparaíso. Casilla 5030, Valparaíso, Chile \\ ${ }^{2}$ Facultad de Farmacia, Universidad de Valparaíso. Casilla 5001, Valparaíso, Chile \\ ${ }^{3}$ Facultad de Ciencias del Mar y de Recursos Naturales, Universidad de Valparaíso. Casilla 5080, Viña del Mar, Chile \\ maria.hidalgo@uv.cl
}

\begin{abstract}
Oxidative stress is the damage at cellular, tissue or organic level produced by reactive oxygen species, that are produced as a result of the exposition to polluting agents, the ingestion through diet, UV radiation or to the oxygen metabolic processes. Chiton granosus is an intertidal organism abundant in Valparaíso, region of Chile. The objective of studying this species, by its abundance, is oriented to its use as biomarkers of oxidative polluting agents and so to evaluate its possible role as a monitoring species of the presence of these polluting agents. To evaluate, gonads, radular and digestive systems were analyzed from Chiton granosus collected from Torpederas, a highly polluted public sector, and Montemar (control area). Two hundreds and fifty nine individuals of Chiton granosus were collected; 133 from Torpederas and 126 from Montemar. The results indicate that no differences due to the sex exists in enzyme activity: catalase in the gonads, superoxide dismutase (SOD) in the radular and digestive systems. No influence of the sampling site was observed, nevertheless the lipoperoxidative damage was concordant with a greater oxidation level in females in the more polluted sector. Total antioxidant capacity exhibited significant differences due to sex and oxidative pollution.
\end{abstract}

Key words: catalase, superoxide dismutase, reactive oxygen species, total antioxidant capacity
Resumen.- El estrés oxidativo es el daño a nivel celular, tisular u orgánico producido por especies reactivas de oxígeno, las que son liberadas al medio interno como resultado de la exposición a contaminantes, la ingestión a través de la dieta, la radiación UV o de los procesos metabólicos que implican oxígeno. El objetivo de este estudio fue evaluar el posible rol de Chiton granosus como especie indicadora de la presencia de contaminantes oxidativos en el intermareal de la región de Valparaíso, Chile central. Para ello, se analizaron parámetros de estrés oxidativo en gónadas y los sistemas radular y digestivo de individuos de Chiton granosus recolectados en la playa Torpederas, sector público, con alto grado de contaminación y caleta Montemar, área de referencia. Se recolectaron 259 ejemplares de Chiton granosus, 133 de playa Torpederas y 126 de caleta Montemar. No se encontraron diferencias debidas al sexo tanto de la actividad de enzima catalasa en gónada, y de superóxido dismutasa (SOD) en los sistemas radular y digestivo, no observándose influencia del sitio de muestreo; sin embargo el daño lipoperoxidativo es concordante con un mayor nivel de oxidación en hembras en el sector más contaminado (Torpederas). La capacidad antioxidante total mostró diferencias en cuanto a sexo y grado de contaminación oxidativo.

Palabras clave: catalasa, superóxido dismutasa, especies reactivas de oxígeno, capacidad antioxidante total

\section{Introducción}

Chiton granosus (Frembly 1889) (Mollusca Polyplacophora) habita paredones rocosos y grietas en el intermareal expuesto al oleaje, con aguas muy oxigenadas (Otaiza \& Santelices 1985). Su distribución es desde Tumbes (Perú) al archipiélago de Chiloé
(Chile) (Forcelli 1999). Es una especie herbívora, presenta sexos separados y es fácil de reconocer entre otros chitones por el color de su gónada. Su recolección es fácil, en el intermareal donde los individuos permanecen expuestos durante la marea baja (Basáez \& Zamorano 1997). Su abundancia y el estado del conocimiento de su biología básica (Kaas \& van Belle 
1985; Moreno \& Jaramillo 1983; Otaíza 1986; Santelices \& Correa 1985) hacen suponer que Chiton granosus sería, una adecuada especie centinela para evaluar la contaminación oxidativa en el litoral marino costero.

Los radicales libres son especies químicas muy reactivas, iones o moléculas, y que poseen uno o más electrones no apareados en sus orbitales externos. En organismos vivos, bajo condiciones normales de metabolismo se forman especies radicalarias a partir de la reducción del oxígeno molecular (Powis \& Southorn 1988). La excesiva producción y/o exposición de un organismo a radicales libres (RL) induce en éste alteraciones biológicas potencialmente conducentes a un daño celular, denominado "estrés oxidativo" (Rodríguez et al. 2001). Los RL más comunes en el organismo son el radical superóxido, el radical hidroxilo y el peroxido de hidrógeno, que aunque no cumple con el requisito de tener electrones desapareados tiene la reactividad necesaria para ser considerado dentro del grupo (Herbert et al. 1994). Este RL anión radical superóxido, puede actuar como agente oxidante en un medio hidrofóbico, como son las membranas celulares y como agente reductor en medio acuoso. A niveles intracelulares son reducidos por dismutación, lo que puede ocurrir espontáneamente o puede ser catalizado por la enzima superóxido dismutasa (SOD). La dismutación del anión radical superóxido produce peróxido de hidrógeno (Goldman \& Welbowan 1992) molécula que puede reaccionar con metales divalentes (libres o unidos a proteínas) actuando como especies prooxidantes generando el radical hidroxilo (reacción de Fenton), considerado el más dañino de los radicales oxigenados. En forma similar, puede reaccionar también con el grupo prostético de metaloproteínas conteniendo hierro (reacción de Haber-Weiss). El radical hidroxilo reacciona a altas velocidades con casi todo tipo de molécula en células vivientes incluyendo los azúcares, aminoácidos, fosfolípidos y bases del ácido desoxirribonucleico (DNA).

En los organismos aerobios existe una gran variedad de sistemas de defensa antioxidante tanto enzimáticos como no enzimáticos, que se coordinan cooperativamente y protegen al organismo de los riesgos que conlleva el estrés oxidativo. Entre ellos destacan las actividades enzimáticas superóxido dismutasa (SOD), glutatión peroxidasa (GPX) y catalasa
(CAT); además del ácido ascórbico (vitamina C), alfatocoferol (vitamina E), glutatión (GSH), beta-caroteno, vitamina A, flavonoides y polifenoles (Hayflick 1985).

La vida en presencia del oxígeno molecular exige contar con una batería múltiple de defensas contra los diversos RL de oxígeno, que por un lado tiendan a impedir su formación y por otro, los neutralicen una vez formados. Para preservar la integridad celular, los organismos aeróbicos disponen de diversos mecanismos de defensa antioxidante, los cuales permiten minimizar los efectos nocivos causados por los agentes oxidantes (Wolf et al. 1986).

Los sistemas antioxidantes se encuentran estratégicamente ubicados en organelos subcelulares para proporcionar la máxima protección y una interacción más adecuada con el compartimento extracelular. Han sido tradicionalmente denominados: defensas no enzimáticas (vitaminas: A, E, C, albúmina, ác. úrico), coenzima Q10 o ubiquinona) (Di Mascio et al. 1991, Bast et al. 1991, Sauberlich 1994, Pal Yu 1994) y defensas enzimáticas (superóxido dismutasa, catalasa y glutatión peroxidasa) (Cotgreave et al. 1988). Las defensas no enzimáticas, interaccionan directamente con radicales libres generados desde el oxígeno, $\left(\mathrm{O}_{2}^{-}\right)$, así como también del producto de su dismutación, mientras las defensas enzimáticas actuarían en la reparación o eliminación de estructuras biológicas dañadas (Pal Yu 1994).

No existe conocimiento acerca de los sistemas antioxidantes en Chiton granosus, ni su relación con el sexo, edad u otra condición, por lo que este estudio aporta los primeros antecedentes acerca de éstos. La posibilidad de utilización de estos organismos como biomarcadores de contaminación ambiental, nos llevó a estudiar su respuesta frente a agentes oxidantes evaluando: el daño lipoperoxidativo, los sistemas de defensas enzimáticas (catalasa y superóxido dismutasa), y no enzimáticas (capacidad antioxidante total), frente a contaminantes oxidativos (metales pesados, principalmente).

Para conocer la biodisponibilidad de los metales pesados reportados en los sectores de muestreo, así como también determinar su influencia en los resultados obtenidos, se determinó la presencia de algunos metales en los sistemas digestivo y muscular de la especie. 


\section{Materiales y métodos}

Se seleccionaron dos sitios ubicados en el intermareal en la costa de la región de Valparaíso, Chile central: la playa Torpederas $\left(33^{\circ} 01^{\prime} \mathrm{S}-71^{\circ} 38^{\prime} \mathrm{W}\right)$, considerada contaminada (CONAMA, datos no publicados) (Tabla 1) y la caleta Montemar (32 $57^{\prime} \mathrm{S}$ - $\left.7^{\circ} 33^{\prime} \mathrm{W}\right)$ que constituye un Area Marina Protegida, a cargo de la Universidad de Valparaíso.

Los ejemplares de Chiton granosus fueron recolectados manualmente en el intermareal rocoso durante marea baja, día por medio, en cada sector, entre abril y junio del año 2000 .

Para determinar las diferencias entre sexos en los mecanismos de defensa antioxidantes, se determinó el sexo de los individuos según el color de las gónadas (Pearse 1979). La gónada, el sistema radular y el digestivo se disecaron y pesaron por separado en una balanza analítica (Scientech SA 120). Se obtuvo el índice gonadosomático, según Lawrence (1970), donde

$$
\mathrm{IGS}=\frac{\text { Peso de la gónada }}{\text { Peso del cuerpo }} \times 100
$$

Los sistemas radular y digestivo fueron colocados separadamente en vasos de precipitado de $50 \mathrm{~mL}$, se les agregó $1 \mathrm{~mL}$ de solución de buffer fosfato salino (PBS $\mathrm{pH} 7,0)$ y $0,5 \mathrm{~mL}$ de detergente Brij (Aldrich), para luego macerar y sonicar por cinco minutos para la liberación de las enzimas (catalasa y superóxido dismutasa) de las células, posteriormente se centrifugó por 10 minutos a $3000 \mathrm{~g}$ y se extrajo el sobrenadante.

El grado de lipoperoxidación se determinó en gónada ya que es el órgano con mayor contenido lipídico en comparación con el estómago y manto (Lawrence 1970). La gónada macerada $(0,5 \mathrm{~g})$ en $1 \mathrm{~mL}$ de buffer $\mathrm{PBS}$ a $\mathrm{pH}=6$ se hizo reaccionar con ácido tricloroacético $(30 \% \mathrm{p} / \mathrm{v})$, se centrifugó y el sobrenadante se hizo reaccionar con ácido tiobarbitúrico $(0,67 \% \mathrm{p} / \mathrm{v})$, se mantuvo en baño de agua a ebullición y posteriormente, una vez frío, se leyó a $\lambda=535 \mathrm{~nm}$ en un espectrofotómetro Cecil 2041. El resultado se interpoló en una curva de calibración de malondialdehído (MDA). Este método se utilizó para cuantificar la magnitud de la peroxidación lipídica, producida por diversas causas, midiendo el producto formado entre los aldehídos generados y el ácido tiobarbitúrico (TBA) (Gutteridge \& Halliwell 1990). Cuando se registró interacción entre los factores sexo y localidad, se definieron los siguientes tratamientos: tratamiento 1, hembras de Montemar; tratamiento 2, hembras de Torpederas; tratamiento 3, machos de Montemar; tratamiento 4, machos de Torpederas.

Para determinar la actividad de catalasa, se pesó la gónada completa y se colocó en un vaso de precipitado de $50 \mathrm{~mL}$, se le agregó $1 \mathrm{~mL}$ de solución buffer fosfato salino (PBS, pH 7,0) y 0,5 mL detergente Brij (Aldrich) luego se maceró y sonicó por cinco minutos para que de esta forma se liberara la catalasa y otras enzimas de las células. Posteriormente, se centrifugó por 10 minutos a $3000 \mathrm{~g}$.

Tabla 1

Principales contaminantes en el agua de Torpederas y Montemar entre 1993 y 1996 (CONAMA datos no publicados)

Main water pollutants from Torpederas and Montemar between 1993 and 1996 (CONAMA unpublished data)

\begin{tabular}{ccccccccc}
\hline Sector/año & $\mathrm{Hg}$ & $\mathrm{Cd}$ & $\mathrm{Pb}$ & $\mathrm{Cu}$ & $\mathrm{Zn}$ & $\mathrm{Cr}$ & $\mathrm{NO}_{2}$ & $\mathrm{NO}_{3}$ \\
\hline Torpederas./93 & 0,353 & 0,36 & 0,36 & 1,23 & 16,33 & 112 & 2,04 & 1,25 \\
Torpederas/94 & 0,172 & 0,253 & 0,743 & 2,397 & 159,47 & 46,00 & 0,014 & 1,348 \\
Torpederas/95 & 0,219 & 0,13 & 0,540 & 15,90 & 110,60 & 62,50 & 0,024 & 2,405 \\
Torpederas/96 & 0,104 & 0,187 & 1,813 & 2,687 & 140,30 & 73,00 & 0,020 & 0,607 \\
Montemar/94 & 0,525 & 0,385 & 1,340 & 3,340 & 40,62 & 51,00 & 0,015 & 0,420 \\
Montemar/95 & 0,089 & 0,12 & 1,255 & 3,460 & 59,53 & 53,00 & 0,022 & 0,455 \\
Montemar/96 & 0,116 & 0,13 & 1,580 & 1,100 & 48,81 & 62,67 & 0,019 & 0,51 \\
\hline
\end{tabular}


La actividad de catalasa se determinó en base a la cinética de degradación de peróxido de hidrógeno, la que es acelerada en presencia de la enzima. La cinética se siguió midiendo la absorbancia a $\lambda=240 \mathrm{~nm}$ en un espectrofotómetro Cecil 2041. Los datos se interpolaron en una curva de calibración realizada a partir de enzima pura (Sigma), haciéndola reaccionar a distintas concentraciones en las mismas condiciones del ensayo con las muestras. Se realizaron 259 ensayos por triplicado y las mediciones se registraron durante un minuto en intervalos de 15 segundos. El proceso se realizó a temperatura ambiente y de la forma más rápida posible para impedir que el macerado sufriera más alteraciones, para evitar la desnaturalización de la enzima, las gónadas fueron extraídas desde ejemplares vivos.

La actividad de superóxido dismutasa (SOD) se determinó en un espectrofotómetro Shimadzu UV-12001 a través de la cinética de reducción del citocromo $\mathrm{C}$ en presencia del sistema xantina-xantina oxidasa, el que produce el anión radical superóxido. Cada órgano se pesó y luego se maceró junto con $1 \mathrm{~mL}$ de buffer fosfato salino (PBS) de $\mathrm{pH}=6,0$, correspondiente al $\mathrm{pH}$ fisiológico del organismo, y $0,5 \mathrm{~mL}$ de detergente Tritón X-100 (Aldrich), para romper las membranas celulares, liberando así la enzima SOD. Posteriormente, se centrifugó durante 10 minutos. La muestra, que se colocó en una celda de cuarzo para ser analizada en el espectrofotómetro, consistió en $2,9 \mathrm{~mL}$ de solución de citocromo $\mathrm{C}(0,023 \% \mathrm{P} / \mathrm{V}), 50 \mu \mathrm{L}$ de solución de xantina oxidasa $(0,25 \% \mathrm{P} / \mathrm{V})$ y $50 \mu \mathrm{L}$ de muestra $(50 \%$ $\mathrm{P} / \mathrm{V})$. El blanco consistió en $3 \mathrm{~mL}$ de solución de citocromo $\mathrm{C}$.

La cinética de formación de citocromo reducido se determinó a partir de los datos de absorbancia a $\lambda=550$ nm. La cinética de la reacción de formación de citocromo reducido se siguió mediante lecturas de absorbancia en un espectrofotómetro a $550 \mathrm{~nm}$, cada 15 segundos, durante 2 minutos. Luego, los datos se interpolaron en una curva de calibración de enzima pura. Los análisis se realizaron por duplicado.

Para determinar la capacidad antioxidante total (TRAP) que consiste en la determinación de defensas antioxidantes no enzimáticas, que actúan atrapando radicales catiónicos ABTS producidos in vitro de la reacción entre el 2,2'-azobis (2-amidino propano (ABAP) y 2,2'-azino-bis (3-etilbenzotiazolina-6sulfónico ácido (ABTS) (Penabad et al. 1994), se disecó el intestino ( $\sin$ ser vaciado) y la gónada de los animales. Una vez pesados, de cada uno de ellos se separó $0,1 \mathrm{~g}$ de tejido. Cada muestra fue tratada por separado. Se maceró el tejido en buffer fosfato salino (PBS) $5 \mathrm{mM}, \mathrm{pH}=6,0$, se centrifugó a 3000 rpm durante 10 minutos y se congeló para su posterior análisis.

Del sobrenadante obtenido por centrifugación se extrajo una fracción de $20 \mu \mathrm{L}$, la que fue depositada directamente en una celda de cuarzo para espectrofotometría de $1 \mathrm{~mL}$ de capacidad; a ésta se le agregó $1 \mathrm{~mL}$ de solución del reactivo 2,2'- azobis(2amidino propano) (ABAP) $10 \mathrm{mM}$ y 2,2'-azino-bis(3etilbenzotiazolina-6-ácido sulfónico) (ABTS) $150^{*} 10^{-6} \mathrm{M}$ (1:1) que había sido incubada por $30 \mathrm{~min}$ a $45^{\circ} \mathrm{C}$ (absorbancia: 0,55, longitud de onda $734 \mathrm{~nm}$, constante). Se siguió la cinética de decaimiento de absorbancia producida por la inactivación ("trapping") de los radicales libres catiónicos generados en la reacción entre ambos, que le confería un tono azulado a la solución; se registraron los valores de absorbancia a $0,10,30$ y 50 segundos, contra un blanco de ABAP (10 $\mathrm{mM}$ ) a una longitud de onda de $\lambda=734 \mathrm{~nm}$. Los datos fueron estandarizados siguiendo el siguiente algoritmo:

\section{(absorbancia t 50 - absorbancia t 10) absorbancia t 30}

El valor obtenido fue interpolado en una curva de calibración utilizando como patrón el antioxidante Trolox, con los que se expresa la capacidad antioxidante total (en adelante TRAP) en $\mu$ moles /L (Romay et al. 1996).

Los tamaños muestreales fueron insuficientes para comparar las medias mediante la prueba $\mathrm{F}$ de ANDEVA, y por esto se aplicaron pruebas noparamétricas. Se utilizó la prueba de Kruskal-Wallis, basada en rangos, para un modelo a un factor, y se definió un factor (tratamiento) que considera cada combinación de niveles de los factores: Grupo 1: Tratamiento 1 sistema muscular (Montemar), Tratamiento 2 sistema digestivo (Montemar). Tratamiento 3 sistema muscular (Torpederas) y Tratamiento 4 sistema digestivo (Torpederas). (Como no hay grupo 2 no debe poner grupo 1 por lo tanto se sugiere eliminar)

Para determinar los metales pesados en los sistemas digestivo y muscular, se digirió el sistema digestivo y la musculatura del pie con una solución de ácido nítricosulfúrico 3:1, durante $24 \mathrm{~h}$ a temperatura ambiente y posteriormente se calentó a $80-95^{\circ} \mathrm{C}$. El carbono presente se eliminó por ebullición con peróxido de 
hidrógeno de 100 volúmenes, hasta que la solución quedó cristalina. Las muestras se filtraron y se analizaron en un espectrofotómetro de absorción atómica Perkin Elmer Analys 300. Los resultados se expresaron en ppm.

En el análisis de las variables estudiadas se usó un análisis de varianza (ANDEVA) de dos factores en un caso (sexo y sector de muestreo) y determinación de parámetros de estrés oxidativo (MDA o TBARS, TRAP, Catalasa y SOD). Se utilizaron pruebas paramétricas y no paramétricas dependiendo de si se cumplían o no los supuestos de normalidad y homocedasticidad de los datos (Draper \& Smith 1981).

\section{Resultados y discusión}

Se recolectaron 259 ejemplares de Chiton granosus: 133 en playa Torpederas y 126 en caleta Montemar; 98 machos entre 4,5 y 6,2 cm (Torpederas); 4,5 a 7,0 cm (Montemar) y 161 hembras entre 3,8 y 5,2 (Torpederas) 4,8 a 7,0 cm (Montemar).

\section{Lipoperoxidación}

El daño lipoperoxidativo, expresado como concentración de malondialdehído (MDA), en el análisis estadístico mostró una interacción entre sexo y sector de muestreo $(P<0,05)$; para el ANDEVA, tanto la prueba $\mathrm{F}$ como la de Kruskal-Wallis detectaron diferencias significativas $(P<0,05)$ entre los tratamientos.

En los resultados de la lipoperoxidación, las hembras de Montemar difieren de los machos de Montemar y de las hembras y machos de Torpederas, también existe diferencia entre hembras de Torpederas y machos de Montemar y entre machos de Montemar y machos de Torpederas. En todas las comparaciones anteriores se obtuvo un valor $P<0,05$.

La mayor lipoperoxidación ocurrió en hembras, en los dos sitios de muestreo, lo que no sería atribuido al contenido lipídico, ya que según Lawrence (1970), es el mismo en ambos sexos. Además, este resultado está en concordancia con la mayor actividad antioxidante de las enzimas catalasa y SOD encontrada en machos, ya que como estas enzimas representan la protección frente al daño peroxidativo, el que su actividad sea mayor indica que la lipoperoxidación debe ser más baja, lo que se muestra en los resultados.

En caleta Montemar, el $90 \%$ de los machos (88 individuos), no presentó daño lipoperoxidativo; las hembras presentaron concentraciones de MDA entre 0,56 y 8,34 nmoles $\mathrm{mL}^{-1}$, con una media de 2,14 $\pm 2,19$ nmoles $\mathrm{mL}^{-1}$ (Tabla 2).

En playa Torpederas, la concentración de MDA en machos resultó notoriamente más baja que en hembras; el daño lipoperoxidativo fue mayor en este sector alcanzando en los machos un máximo de 0,65 nmoles $\mathrm{mL}^{-1}$ de MDA, y en las hembras 8,78 nmoles $\mathrm{mL}^{-1}$ de MDA; en caleta Montemar también se encontraron valores superiores de concentración de MDA en las hembras por sobre los machos (valor máximo 8,34 nmoles $\mathrm{mL}^{-1}$ y 0,10 nmoles $\mathrm{mL}^{-1}$ respectivamente). No se encontró relación entre la concentración de MDA y el IGS para ninguna de las dos poblaciones analizadas. El daño oxidativo resultó ser independiente de la longitud total (como posible estimación de la edad), ya que aunque ésta fue similar en todos los casos, la lipoperoxidación fue mayor en las hembras de ambos sectores (Tabla 2).

Tabla 2

Relación entre la concentración (promedio \pm DE) de malondialdehído (MDA) y la longitud total (promedio \pm DE) de los ejemplares de C. granosus recolectados en Torpederas y Montemar

Relation between the concentration (mean \pm SD) of Malondialdehyde (MDA) and the total length (mean \pm SD) in C. granosus specimens collected in Torpederas and Montemar

\begin{tabular}{lcccc}
\hline \multirow{2}{*}{ Parámetro } & \multicolumn{2}{c}{ Torpederas } & \multicolumn{2}{c}{ Montemar } \\
& Hembras $(\mathrm{n}=18)$ & Machos $(\mathrm{n}=22)$ & Hembras $(\mathrm{n}=18)$ & Machos $(\mathrm{n}=22)$ \\
\hline Longitud total $(\mathrm{cm})$ & $5,41 \pm 0,07$ & $5,35 \pm 0,11$ & $5,53 \pm 0,97$ & $5,49 \pm 0,53$ \\
MDA $\left(\mathrm{nm} \mathrm{mL}^{-1}\right)$ & $2,70 \pm 2,33$ & $0,22 \pm 0,18$ & $2,14 \pm 2,19$ & $0,054 \pm 0,014$ \\
\hline
\end{tabular}




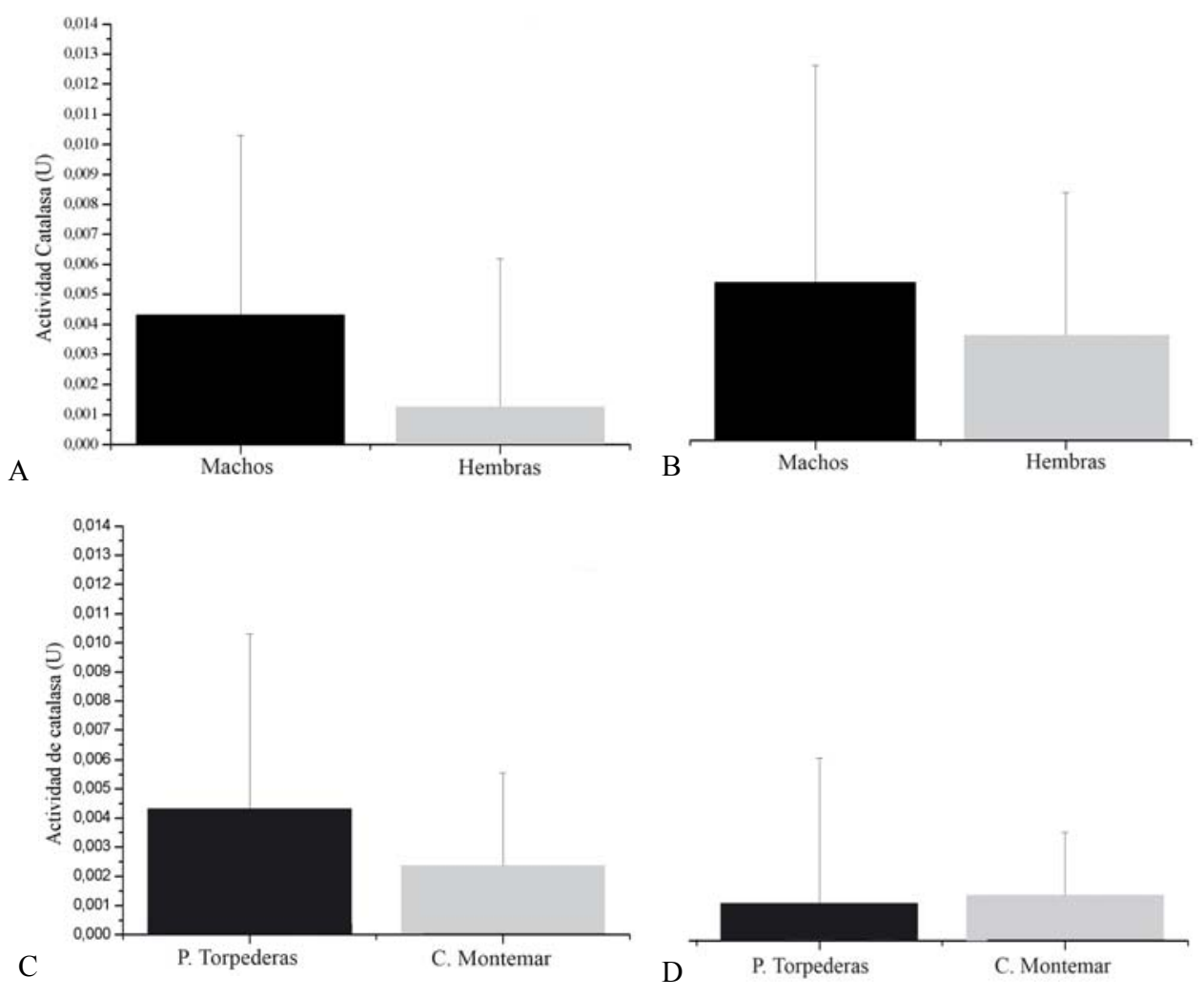

Figura 1

Comparación de la actividad enzimática de catalasa en gónadas masculinas y femeninas de ejemplares de $C$. granosus, provenientes de Torpederas y Montemar (promedio y desviación estándar): (A) machos y hembras de Torpederas (n=41), (B) machos y hembras de Montemar ( $n=38)$. (C) machos de Torpederas y Montemar $(n=45)$. (D) hembras de Torpederas y Montemar (n= 34). (n= número muestral)

Comparison of male and female gonadal catalase enzymatic activity from C. granosus from Torpederas and Montemar (mean and standard deviation): (A) males and females from Torpederas $(n=41)$. (B) males and females from Montemar $(n=38)$. (C) males from Torpederas.and Montemar $(n=45)$. (D) females from Torpederas and Montemar $(n=34)$. $(n=$ number of sampled gonads)

El mayor nivel de daño oxidativo en hembras, al parecer no se debería a diferencias en la concentración lipídica de las gónadas ya que Lawrence (1970) comprobó en Chiton tuberculatus, en Costa Rica, que tanto ovarios como testículos poseen igual cantidad de lípidos totales. El IGS es un indicador de la madurez de la gónada, por lo que se esperaría, basándose en la teoría del envejecimiento por radicales libres (Hayflick 1985), que individuos con mayor grado de madurez sexual presentaran mayor grado de lipoperoxidación, al igual que individuos de mayor edad (longitud total mayor), lo que no se comprobó.

La zona de muestreo resultó ser un factor determinante en el grado de lipoperoxidación encontrado ya que tanto machos como hembras de playa Torpederas presentaron mayor daño que los de caleta Montemar, lo que coincide con la mayor cantidad de contaminantes en la primera (Tabla 1).

\section{Actividad de catalasa}

La actividad de la catalasa en machos $\left(4,35 \times 10^{-3} \pm\right.$ $0,0068 \mathrm{U})$ de Chiton granosus fue superior a la de hembras $\left(8,07 \times 10^{-4} \pm 0,00065 \mathrm{U}\right)$ de Torpederas. Los resultados en machos y hembras de Montemar fueron $3,13 \times 10^{-3} \pm 0,00641 \mathrm{U}, \mathrm{y} 1,36 \times 10^{-3} \pm 0,0013 \mathrm{U}$ respectivamente (Fig. 1). No se encontraron diferencias significativas entre la actividad de catalasa y el sexo y el sector combinados (Kruskal-Wallis, $P=0,516$ ). 


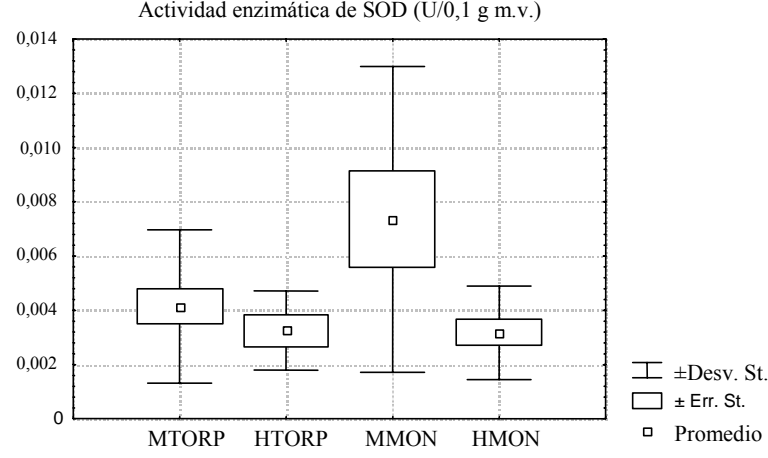

Figura 2

Actividad enzimática de superóxido dismutasa (U/0,1 g de masa visceral) en C. granosus. MTORP: machos de

Torpederas; HTORP: hembras de Torpederas; MMONT: machos de Montemar; HMONT: hembras de Montemar. (prueba $\mathrm{T}$ y prueba Wilcoxon)

Superoxide dismutase enzymatic activity (U/0,1 g visceral mass) in C. granosus. MTORP: males from Torpederas; HTORP: females from Torpederas; MMONT: males from Montemar; HMONT: females from Montemar. (Wilcoxon and $t$ test).

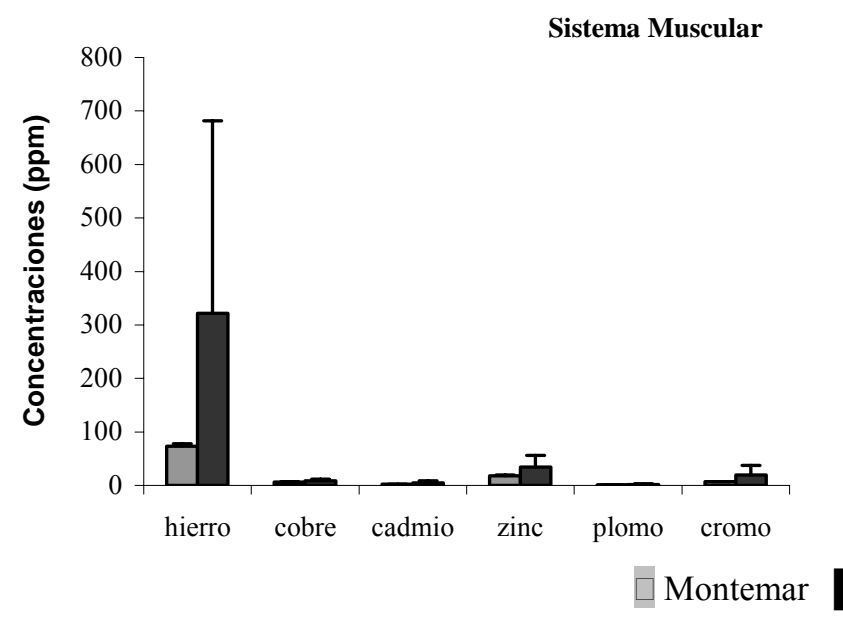

\section{Actividad de superóxido dismutasa}

No se encontró actividad enzimática ni en pie ni en gónada de Chiton granosus ya que no se observó variación en la velocidad de reducción del citocromo $\mathrm{C}$. En la masa visceral, el promedio de la actividad enzimática en machos de Montemar fue de 0,0073 \pm $0,0056 \mathrm{U} / 0,1 \mathrm{~g}$ de masa visceral en tanto que en hembras del mismo sector fue de 0,0032 $\pm 0,0017$ $\mathrm{U} / 0,1 \mathrm{~g}$ de masa visceral. En Torpederas, el promedio de la actividad enzimática de machos fue de 0,0041 \pm $0,0028 \mathrm{U} / 0,1 \mathrm{~g}$ de masa visceral y el de hembras fue de $0,0032 \pm 0,0014 \mathrm{U} / 0,1 \mathrm{~g}$ de masa visceral. (Fig. 2). En ambos sectores, los machos mostraron una mayor actividad de SOD que las hembras.

No se encontraron diferencias significativas entre las combinaciones de niveles de los factores sexo y sector $(P=0,229)$. No se encontró relación entre la masa visceral y la actividad enzimática, tampoco entre el tamaño del individuo y su masa visceral; factores como cantidad y accesibilidad al alimento pueden ser los causantes de dichas diferencias. Los resultados son acordes con el nivel de lipoperoxidación y actividad de la enzima catalasa, sin embargo, el factor localidad no mostró diferencias significativas en este parámetro.

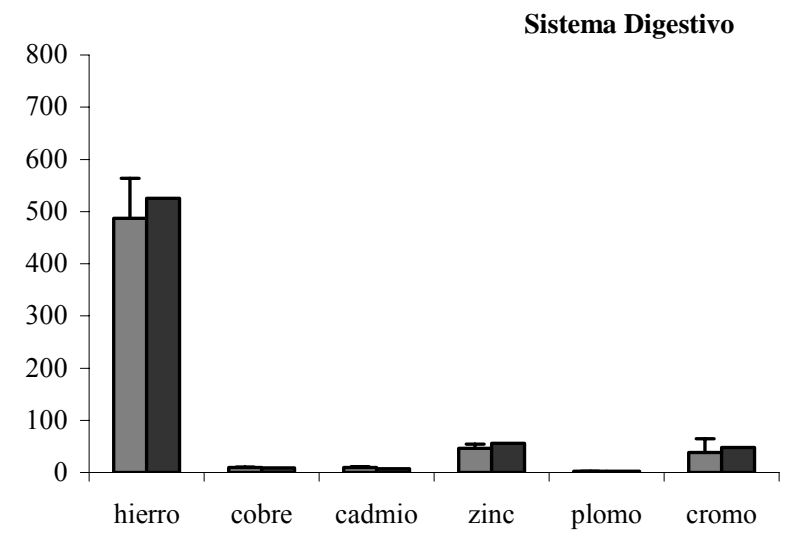

Figura 3

Concentración (ppm) de metales pesados en ejemplares de C. granosus de Torpederas y Montemar.

A. Sistema muscular, B. Sistema digestivo

Heavy metal concentration (ppm) in C. granosus specimens from Torpederas and Montemar.

A. Muscular system, B. Digestive system 


\section{Capacidad antioxidante total en plasma (TRAP)}

Se realizó una prueba de normalidad KolmogorovSmirnov de la variable TRAP determinada en tejido digestivo y gonádico. De acuerdo a los resultados obtenidos, $P=0,59$ para la variable Trap Dig y $P=0,711$ para la variable Trap Gon, a un nivel de significación ( $\alpha$ ) del 5\%, no existe evidencia significativa para suponer que las variables no se distribuyan de acuerdo a un modelo normal.

La prueba de Levene (homogeneidad de varianzas) proporcionó un $P=0,022$ para la variable Trap Gon y $P=0,163$ para la variable Trap Dig. De acuerdo a estos valores, no existe evidencia significativa $(\alpha=5 \%)$ para suponer heterocedasticidad de la variable Trap Dig. En el caso de la variable Trap Gon, su significatividad es $<5 \%$, sin embargo, no es lo suficientemente pequeña como para pensar que la robustez del estadístico $\mathrm{F}$ del ANDEVA, inhabilite su aplicación para la comparación de medias.

Considerando lo anterior, para analizar el efecto de los factores sexo y del sector, sobre las variables bajo estudio, se utilizó la prueba $\mathrm{F}$ de ANDEVA, detectándose un efecto de interacción altamente significativo $(P=0,001)$, lo cual impide detectar los efectos de los factores en forma separada. El análisis de varianza mostró un efecto significativo del factor TRAT (sexo y sector) $(P=0,012)$.

Para detectar los tratamientos que presentaban diferencias se aplicó la prueba de comparaciones múltiples LSD (mínima diferencia significativa), que evidenció la formación de dos grupos homogéneos, uno formado por tratamientos sin diferencias en sus efectos sobre la variable en estudio, y otro grupo formado por tratamientos significativamente diferentes. El grupo 1 conformado por el tratamiento 1 (hembras de Torpederas) y el tratamiento 4 (machos de Montemar) y el grupo 2, por el tratamiento 2 (machos de Torpederas) y el tratamiento 3 (hembras de Montemar).

En el análisis de TRAP digestivo no se encontraron diferencias significativas $(P>0,05)$ ni del efecto de interacción como de los efectos principales de los factores sector y sexo. Se encontraron diferencias significativas en la respuesta antioxidante en tejido gonádico, entre sexos en ambos sectores y entre individuos del mismo sexo, en sectores diferentes, lo que coincide con los resultados encontrados en el daño a lípidos (TBARS o MDA); sin embargo, no se presentaron diferencias significativas en la capacidad antioxidante en tejido digestivo.

La alta TRAP del tejido digestivo puede deberse al contenido del mismo ya que estos animales se alimentan principalmente de algas que ramonean la superficie del sustrato rocoso en que viven (Otaíza 1986, Santelices \& Correa 1985). La varianza muy elevada también es explicable por el diferente contenido de los intestinos examinados, descartando la influencia del peso, ya que se trabajó con la misma masa en gramos. Por lo anterior, la TRAP del tejido gonádico sería más reveladora de la condición propia del animal; sin embargo, la varianza encontrada resulta difícil de explicar, ya que todos los individuos recolectados poseían gónadas aparentemente maduras (turgentes). No existen publicaciones en chitones $\mathrm{u}$ otros moluscos que permitan comparar nuestros resultados respecto de los diferentes parámetros de estrés oxidativo determinados en este primer estudio, en Chiton granosus; es así que sólo hay un estudio en relación a parámetros de estrés oxidativo y especies marinas (Huerta et al. 2000).

Con respecto de los metales pesados determinados en los sistemas digestivo y muscular de Ch. granosus, se encontró una alta acumulación de hierro en los individuos de ambos sectores de muestreo siendo mayor en el sistema digestivo, aún cuando no hubo relación entre la masa y la concentración de metales (Tabla 3). La masa del sistema muscular duplicó y en ocasiones triplicó la masa del sistema digestivo, por lo que se asume que el sistema digestivo por su contenido, presentaba una mayor acumulación. Los hábitos alimenticios de Ch. granosus y su desplazamiento lento (como para escapar de los agentes químicos) hacen de ésta una especie susceptible a agentes contaminantes, como los metales pesados.

No se encontraron diferencias significativas entre los efectos de los diferentes tratamientos sobre la media de las variables bajo estudio: hierro- $P=0,277$; cobre$P=0,142$; cadmio- $P=0,155$; zinc- $P=0,087$; plomo$P=0,277$ y cromo- $P=0,490$. En consecuencia, los contaminantes evaluados no son indicativos del grado de contaminación de los individuos en los sectores en estudio (Fig. 3), lo que podría explicar la escasa diferencia entre los parámetros de estrés oxidativo evaluados como defensas enzimáticas, aún más, conociéndose el rol que el hierro cumple en los procesos oxidativos como generador de especies reactivas de oxígeno (Herbert et al. 1994). 
Tabla 3

Capacidad antioxidante total (TRAP) (promedio \pm DE) en los sistemas gonádico y digestivo de C. granosus

Total antioxidant capacity (TRAP) (mean \pm SD) in gonadal and digestive systems of $C$. granosus

\begin{tabular}{|c|c|c|c|c|}
\hline \multirow{3}{*}{$\begin{array}{l}\text { Parámetro } \\
\text { sector / sexo }\end{array}$} & \multicolumn{4}{|c|}{ TRAP $\mu$ moles $L^{-1}$} \\
\hline & \multicolumn{2}{|c|}{ Gónada } & \multicolumn{2}{|c|}{ Digestivo } \\
\hline & $\operatorname{Machos}(n=29)$ & Hembras $(n=22)$ & $\operatorname{Machos}(n=29)$ & Hembras $(n=23)$ \\
\hline Torpederas & $2,12 \pm 0,033$ & $0,65 \pm 0,033$ & $4,25 \pm 0,12$ & $1,89 \pm 0,074$ \\
\hline Montemar & $0,77 \pm 0.051$ & $1,77 \pm 0,078$ & $2,38 \pm 0,22$ & $3,15 \pm 0,24$ \\
\hline
\end{tabular}

Muchos invertebrados marinos producen especies reactivas de oxígeno (ERO) (Lesser 2006); los moluscos bivalvos producen ERO en respuesta a los xenobióticos (Winston et al. 1990) y también debido a cambios de temperatura (choque térmico) (Abele et al. 2002). Las ERO son también importantes en la respuesta celular inmune mediada de moluscos ante patógenos procariota y eucariota (Adema et al. 1991). Muchos moluscos bivalvos son eurioxicos y sobreviven fluctuaciones entre hipoxia/anoxia y normoxia con cada ciclo de marea; durante las transiciones anoxia-normoxia, las especies eurioxicas producen ERO lo que conduce a un estado de estrés oxidativo (Dykens \& Shick 1988). Los artrópodos marinos varían sus defensas antioxidantes de acuerdo a su nivel de metabolismo aeróbico, exposición a medios ambientes fríos o exposición a radiación ultravioleta (Maciel et al. 2004, Camus \& Gulliksen 2005, Gouveia et al. 2005); sorprendentemente, los artrópodos marinos pierden superóxido dismutasa (SOD) $\mathrm{Cu} / \mathrm{Zn}$ (Brouwer et al. 1997, 2003), pero estos animales usan una hemocianina dependiente de cobre para el transporte de oxígeno y presentan una inusual SOD Mn citosólica (Brouwer et al. 2003). Estudios de estrés oxidativo también se han realizado en equinodermos, vertebrados y fotoautótrofos (Lesser 2006).

En resumen, Chiton granosus no presenta diferencias significativas en la actividad de enzimas antioxidantes (catalasa y superóxido dismutasa) entre machos y hembras. Sin embargo, no existe concordancia entre los resultados obtenidos para el parámetro que da cuenta de las defensas no enzimáticas (TRAP) y aquellos presentados para otros parámetros de la respuesta antioxidante o del daño por ROS en estos organismos. El factor ambiental oxidativo se manifiesta en el grado de lipoperoxidación, existiendo mayor daño oxidativo en lípidos encontrado en hembras y en el estado de defensas antioxidantes no enzimáticas
(TRAP) evaluado en tejido gonádico. La acumulación de metales en los organismos, en su mayoría hierro, fue similar en ambos sectores de muestreo. Las diferencias encontradas en los parámetros evaluados, debidas al sexo, indicarían que los procesos metabólicos y bioquímicos de adaptación a los procesos oxidativos generados por agentes ambientales oxidantes, serían diferentes entre machos y hembras.

Estos resultados constituyen las primeras evidencias de los sistemas antioxidantes de Chiton granosus, así como del daño lipoperoxidativo relacionado con la contaminación ambiental, en particular por agentes oxidantes, entre los que se cuentan los metales pesados.

\section{Agradecimientos}

A CONAMA por la información acerca de los contaminantes presentes en las zonas de muestreo, al Prof. Luis López de la Facultad de Ciencias de la Universidad de Valparaíso, por su asesoría en la determinación de metales pesados. A los revisores del presente manuscrito por sus aportes.

\section{Literatura citada}

Abele D, K Heise, H Pörtner \& S Puntarulo. 2002. Temperature-dependence of mitochondrial function and production of reactive oxygen species in the intertidal mud clam Mya arenaria. Journal of Experimental Biology 205: 1831-41.

Adema CM, WP Van der Knaap \& T Sminia. 1991. Molluscan hemocytemediated cytotoxicity: the role of reactive oxygen intermediates. Reviews in Aquatic Sciences 4: 201-23. 
Bast A, GR Haenen \& CJ Doelman. 1991. Oxidants and antioxidants: State of the Art. American Journal of Medicine. 91(suppl. 3C): 2-13.

Basáez F \& A Zamorano. 1997. Estudio comparativo de la espermatogenesis de tres especies de moluscos poliplacóforos chilenos: Acanthopleura echinata (Barnes, 1823), Chiton granosus (Frembly, 1827) y Chaetopleura peruviana (Lamarck, 1819). Tesis de Pedagogía en Biología y Ciencias, Universidad de Playa Ancha de Ciencias de la Educación, 89 pp.

Brouwer M, TH Brouwer, W Grater, JJ Enghild \& IB Thogersen. 1997. The paradigm that all oxygen-respiring eukaryotes have cytolosolic CuZn-suproxide dismutase and that Mn-superoxide dismutase is localized to mitochondria does not apply to a large group of marine arthropods. Biochemistry 36: 13381-88.

Brouwer M, TH Brouwer, W Grater \& N Brown-Peterson. 2003. Replacement of a cytosolic copper/zinc superoxide dismutase by a novel cytosolic manganese superoxide dismutase in crustaceans that use copper (haemocyanin) for oxygen transport. Journal of Biochemistry 374: 21928.

Camus L \& B Gulliksen. 2005. Antioxidant defense properties of Arctic amphipods: comparison between deep-, sublittoral and surface species. Marine Biology 146: 355-62.

Cotgreave I, P Moldeus \& S Orrenius. 1988. Host biochemical defense mechanisms against prooxidants. Annual Review of Pharmacology and Toxicology 28: 189212.

Di Mascio P, ME Murphy \& H Sies. 1991. Antioxidant defense systems: The rol of carotenoids, tocopherols, and thiols. The American Journal of Clinical Nutrition 53: 194S-200S.

Draper N \& H Smith. 1981. Applied Regression Analysis, 709 pp. $2^{\text {nd }}$ Ed. John Wiley \& Sons, USA.

Dykens JA \& JM Shick 1988. Relevance of purine catabolism to hypoxia and recovery in euryoxic and stenoxic marine invertebrates, particularly bivalve molluscs. Comparative Physiology and Biochemistry $C$ 91: 35-41.

Gouveia GR, DS Marques, BP Cruz, LA Geracitano \& LE Nery. 2005. Antioxidant defenses and DNA damage induced by UV-A and UV-B radiation in the crab Chasmagnathus granulata (Decapoda, Brachyura). Photochemistry \& Photobiology 81: 398-403.
Greenwald R. 1990. Current approaches to the development of oxygen radical scavengers. Drugs of Today 26(5): 299307.

Forcelli D. 1999. Moluscos magallánicos. Guía de moluscos de la Patagonia y el sur de Chile. Parte II. 200 pp. Total Austral S.A. Argentina.

Goldman G \& R Welbowan. 1992. Oxygen free radicals are required for ischemia-induced leukotriene B4 diapedesis. Surgery 111 (3): 287-93.

Gutteridge JM \& B Halliwell. 1990. The measurement and mechanism of lipid peroxidation in biological systems. Trends in Biochemical Sciences 15: 129-135.

Hayflick L. 1985. Theories of biological aging. Experimental Gerontology 20: 145-149.

Herbert V, S Shaw, E Jayatilleke \& T Stopler-Kasdan. 1994. Most free-radical injury is iron-related: it is promoted by iron, hemin, holoferritin and Vitamin C, and inhibited by desferoxamine and apoferritin. Stem Cells 12: 289-303.

Huerta D, R Cifuentes \& M Fonseca. 2000. El "erizo de mar” Tetrapygus niger como marcador biológico de estrés oxidativo. Revista Peruana de Biología 7(2): 198-201.

Kaas P \& R van Belle. 1985. Monography of living chitons, 400 pp. Spring-Verlag, Berlín.

Lawrence J. 1970. The lipid composition of the organs of two species of tropical chitons. Carribean Journal of Science 10: $1-3$.

Maciel FE, CE Rosa, EA Santos, JM Monserrat \& LE Nery. 2004. Daily variations in oxygen consumption, antioxidant defenses, and lipid peroxidation in the gills and hepatopancreas of an estuarine crab. Canadian Journal of Zoology 82: 1871-77.

Moreno CA \& E Jaramillo. 1983. The role of grazers in the zonation of intertidal macroalgae of the Chilean coast. Oikos 41: 73-76.

Otaiza RD. 1986. Patrones de distribución vertical de chitones y efecto de pastoreo de Chiton granosus en roqueríos intermareales de Chile central. En: Santelices B (ed), Simposio Internacional. Usos y funciones ecológicas de las algas marinas bentónicas. Monografías Biológicas 4: $173-190$

Otaiza RD \& B Santelices. 1985. Vertical distribution of chitons (Mollusca: Polyplacophora) in the rocky intertidal zone of central Chile. Journal of Experimental Marine Biology and Ecology 86: 229-240. 
PalYu B. 1994. Cellular defenses against damage from reactive oxygen species. Physiolical Reviews 73(1): 139162 .

Pearse J. 1979. Polyplacophora. En: Giese A \& J Pearse (eds), Reproduction of marine invertebrates 5: 27-85. Academic Press, Nueva York.

Penabad C, I Conde \& P Marqui. 1994. Total free radicaltrapping capacity of serum from diabetics. Clinical Chemistry 40(11): 2116-2117.

Powis G \& P Southorn. 1988. Free radicals in medicine I: chemical nature and biological reactions. Mayo Clinic Proceedings 63: 381-389.

Rodríguez J, J Menéndez \& Y Trujillo. 2001. Radicales libres en la biomedicina y estrés oxidativo. Revista Cubana de Medicina Militar 30(1): 36-44.

Romay C, C Pascual \& E A Lissi. 1996. The reaction between ABTS radical cation and antioxidants and it's use to evaluate the antioxidant status of serum samples.
Brazilian Journal of Medical and Biological Research 29(2): 175-183.

Santelices B \& J Correa. 1985. Differential survival of macroalgae to digestion by intertidal herbivore molluscs. Journal of Experimental Marine Biology and Ecology 88: 183-191.

Sauberlich H. 1994. Pharmacology of vitamin C. Annual Review of Nutrition 14: 371-391.

Stuardo J. 1959. Ensayo de una clave para familias y géneros chilenos de Polyplacophora con generalidades del grupo e inclusión de algunas especies. Investigaciones Zoológicas Chilenas 5: 139-148.

Winston GW, DR Livingstone \& F Lips. 1990. Oxygen reduction metabolism by the digestive gland of the common marine mussel, Mytilus edulis L. Journal of Experimental Zoology 255: 296-308.

Wolf S, A Barrer \& RT Dean. 1986. Free radicals, lipid and protein degradation. Trends in Biochemical Sciences 11: 27-31.

Recibido el 30 de septiembre de 2005 y aceptado el 20 de junio de 2006 